\title{
Exploring the Intersections of Education and Healthcare Delivery Using a Clinical Learning Environment Framework
}

\author{
Bridget C. O'Brien, $P h D^{7}$ and Darcy A. Reed, MD MPH ${ }^{2}$ \\ 'Department of Medicine, University of California, San Francisco, San Francisco, CA, USA; ${ }^{2}$ Department of Medicine, Mayo Clinic College of \\ Medicine and Science, Rochester, MN, USA.
}

J Gen Intern Med 34(5):654-6

DOI: $10.1007 / \mathrm{s} 11606-019-04929-4$

(c) Society of General Internal Medicine 2019

\begin{abstract}
A midst rapid changes in the healthcare system, academic health centers are paying greater attention to the intersection of education and healthcare delivery. Various reports and articles urge health systems leaders, administrators, and educators to seize opportunities to align the purpose, goals, mission, and priorities of medical education with those of healthcare delivery to achieve better outcomes for both patients and learners. ${ }^{1-5}$ Proposed alignment strategies run the gamut from defining shared performance standards to innovative design of systems, programs, and processes that support mutual goals such as high-value care, patient safety, and quality learning environments. ${ }^{1-3,6}$

The need for alignment resonates with both educators and health systems leaders who jostle competing priorities within the healthcare system on a daily basis. And yet, it is a bit perplexing that two, obviously intertwined, elements of the healthcare system-delivery and education - could drift in such different directions that they are often described as two distinct systems. Bridging roles have been proposed as a way to increase communication between education and delivery system leadership. ${ }^{1}$ Similarly, we might think about bridging roles for scholarship. Most authors and journals target a single audience such as educators, system improvement experts, or leaders in healthcare delivery, but the Journal of General Internal Medicine has audience with all three and thus can bridge the gap. Although this is a special issue on education, several articles in the issue present opportunities to think more deeply about alignment and synergies between education and other elements of the healthcare system. While the articles in this issue rightfully focus on education, we use this commentary to highlight ways in which scholarship can bridge education and healthcare delivery systems. We do this by examining the articles in relation to the boundary-spanning framework of clinical learning environments.
\end{abstract}

Published online April 16, 2019

\section{CLINICAL LEARNING ENVIRONMENT}

The term clinical learning environment, popularized by the Accreditation Council for Graduate Medical Education, encapsulates both the healthcare delivery system (clinical) and the education system (learning) in one construct. The term environment captures the notion of a dynamic system in which many elements interact. A recent report published by the Macy Foundation characterized the learning environment as "the social interactions, organizational cultures and structures, and physical and virtual spaces that surround and shape participants' experiences, perceptions, and learning." ${ }^{7}$ Based on this multi-dimensional characterization, many activities currently framed as education or delivery system improvement could be rigorously analyzed through a clinical learning environment framework to incorporate both health delivery systems and education. For example, in clinical settings, an analysis of the learning environment could call attention to space constraints or staffing shortages that impact both patients' and trainees' experiences of a clinical setting.

While none of the articles in this section used clinical learning environment as an explicit framing, several describe changes in one or more elements of the clinical learning environment that ripple through other elements. For example, two papers recognized changes in the composition and structure of the workforce as important sources of learning opportunities. Gottenborg and colleagues appreciated the growing reliance on NPs and PAs to manage the clinical workload on inpatient medicine services, the lack of interaction between these health professionals and medicine residents, and the opportunity to design a new organizational structure (an integrated general medicine ward team comprised of medicine residents and NP or PA fellows). ${ }^{8}$ In this paper, the authors oriented the objectives, evaluation, and discussion of the new interprofessional team toward educational outcomes and implications. A clinical learning environment framing could introduce the additional perspective of outcomes and implications for the clinical service thereby opening up a scholarly discourse about alignment between education and health delivery systems. Wang and colleagues identified the emerging "triagist" role as an underutilized opportunity to purposefully align the goals of healthcare delivery and education. ${ }^{9}$ Although the role was primarily created to improve delivery system performance metrics such as appropriate use of 
hospital resources and efficient flow of patients from the emergency department to inpatient services, the authors recognized an untapped and potentially high-value learning opportunity for residents if some of the work of triagists could be shifted from staff physicians to trainees and structured to provide sufficient support and supervision. Wang and colleagues implicitly illustrate the value of a clinical learning environment analysis that ties together clinical service goals and educational goals.

Scholarly conversations about alignment of medical education and healthcare delivery can also begin with analyses of existing educational structures within clinical learning environments. Pincavage and colleagues examined the current structure of internal medicine clerkships via a national survey of clerkship directors. ${ }^{10}$ The results revealed that over half of internal medicine clerkships do not require ambulatory training components. This raises important questions about the alignment, or lack thereof, of the clinical learning environments of medical student internal medicine clerkship education (inpatient predominant) and the likely skills and experiences needed to effectively deliver care in current and future complex health delivery systems. Similarly, DeWaters and colleagues reviewed the literature on ambulatory block structures in internal medicine residency programs to identify the impact of these new educational structures on what could be called the components of the clinical learning environment-namely perceptions of the experience (resident and patient satisfaction), organizational structures (conflict between inpatient and outpatient responsibilities, continuity of care), and learning/clinical outcomes (patient health outcomes). ${ }^{11}$ The findings raise intriguing questions about the value of ambulatory blocks as clinical learning environments.

Though often taken for granted, material aspects of clinical learning environments play a key role in processes of care and learning opportunities. Wallach and colleagues studied one of the more prominent, and widely contested, material components of the clinical learning environment - the electronic health record. ${ }^{12}$ From a delivery system standpoint, restricting medical students' access to the EHR might be beneficial for efficiency, financial, and liability purposes, but this short-term view has consequences for longer-term workforce development and patient safety. The authors astutely point to a recent change in Centers for Medicare \& Medicaid Services (CMS) policy as an opportunity for further scholarship on student learning experiences. Such changes cause perturbations in the clinical learning environment that open up new possibilities for thoughtful design of activities to support both learning and delivery system needs.

Perhaps the greatest challenge for scholarship that links education and delivery systems is the choice of appropriate performance metrics and data as indicators of core values. Stratton raises our awareness of a common tendency to let measures and metrics divert our attention toward quantifiable, surface characteristics and away from deeper questions about quality. ${ }^{13}$ The quest to rationalize the educational process prioritizes consistency, efficiency, and "objective" data. We see this tendency in both medical education and in healthcare delivery. As a case in point, we noticed that most articles in this theme of the issue cite accreditation or competency standards in their introductory paragraph, presumably as a way to legitimize and rationalize their educational initiatives and goals. Stratton's article reminds us that adherence to data collection protocols and compliance with standards, while enticing goals, alone fail to ensure quality and improvement. His approach to continuous quality improvement in an educational program seems well suited to the dynamic, interactive nature of a clinical learning environment. This approach encourages consideration of multiple components of the healthcare system, with monitoring to ensure allegiance to core values and to avoid redirection of improvement efforts "toward other institutional ends."

\section{CLOSING REMARKS}

Although this special issue of JGIM shines a light on educational scholarship, several of the included articles also illuminate prospects for subsequent exploration of synergies between education and other components of the healthcare system. We offered clinical learning environments as a potential framework to guide such work. The clinical learning environment framework draws attention to ways in which components of the healthcare system interact with and impact one another. In this respect, it offers a helpful construct within which to critically examine processes, identify discordant elements, and prompt discussion among key stakeholders about interventions to optimize both education and healthcare delivery systems.

Corresponding Author: Bridget C. O'Brien, PhD; Department of Medicine University of California, San Francisco, 533 Parnassus Ave. Suite U-80, San Francisco, CA 94143-0710, USA (e-mail: bridget. obrien@ucsf.edu).

Publisher's Note: Springer Nature remains neutral with regard to jurisdictional claims in published maps and institutional affiliations.

\section{REFERENCES}

1. Gupta R, Sehgal N, Arora VM. Aligning Delivery System and Training Missions in Academic Medical Centers to Promote High-Value Care. Academic Medicine. 2018.

2. Sklar DP, Hemmer PA, Durning SJ. Medical Education and Health Care Delivery: A Call to Better Align Goals and Purposes. Academic Medicine. 2018; 93(3):384-90.

3. Wagner R, Newton RC, Casey BR, Koh NJ, Weiss KB. The Overview of the CLER Program: CLER National Report of Findings JGME. 2018;2.

4. Skochelak SE. A decade of reports calling for change in medical education: what do they say? Acad Med. 2010;85(9 Suppl):S26-33.

5. Gonzalo JD, Haidet P, Papp KK, Wolpaw DR, Moser E, Wittenstein RD, Wolpaw T. Educating for the 21st-Century Health Care System: An Interdependent Framework of Basic, Clinical, and Systems Sciences. Acad Med. 2017;92(1):35-39.

6. Gonzalo JD, Lucey C, Wolpaw T, Chang A. Value-Added Clinica Systems Learning Roles for Medical Students That Transform Education 
and Health: A Guide for Building Partnerships Between Medical Schools and Health Systems. Acad Med. 2017;92(5):602-607.

7. Irby DM. Improving environments for learning in the health professions. Proceedings of a conference sponsored by Josiah Macy Jr. Foundation in April 2018; New York, NY: Josiah Macy Jr. Foundation, 2018.

8. Gottenborg $\mathbf{E}$, Limes $\mathbf{J}$, Olson A, Wolfe B. Learning Together: Integration of Advanced Practice Providers into a Traditional Ward Team Model. J Gen Intern Med https://doi.org/10.1007/s11606-019-04880-4

9. Wang E, Velásquez ST, Smith CJ, Matthias TH, Schmit D, Hsu S, Leykum LK. Triaging Inpatient Admissions: An Opportunity for Resident Education. J Gen Intern Med https://doi.org/10.1007/s11606-01904882-2.

10. Pincavage A, Fagan M, Osman NY, Leizman DS, DeWaay D, Curren C, Ismail N, Szauter $\mathbf{K}$, Kisielewski M, Shaheen AW. One Structure does not fit all: A National Survey of Undergraduate Clinical Education in
Internal Medicine. J Gen Intern Med https://doi.org/10.1007/s11606019-04892-0.

11. DeWaters A, Loria $\mathbf{H}$, Mayo $\mathbf{H}$, Chisty A, Nguyen OK. The Impact of Block Ambulatory Scheduling on Internal Medicine Residencies: A Systematic Review. J Gen Intern Med https://doi.org/10.1007/s11606019-04887-x.

12. Wallach P, Foster LM, Cuddy MM, Hammoud MM, Holtzman KZ, Swanson DB. Electronic Health Record Use in Internal Medicine Clerkships and Sub-internships for Medical Students Graduating from 2012 to 2016. J Gen Intern Med https://doi.org/10.1007/s11606-01904902-1.

13. Stratton T. Legitimizing Continuous Quality Improvement (CQI) Efforts: Navigating Rationalization in Medical Education. J Gen Intern Med https://doi.org/10.1007/s1 1606-019-04875-1. 